\title{
Édouard Glissant, Philosophie de la Relation
}

\section{Luigia Pattano}

\section{(2) OpenEdition \\ 10 Journals}

\section{Edizione digitale}

URL: http://journals.openedition.org/studifrancesi/7402

DOI: $10.4000 /$ studifrancesi.7402

ISSN: 2421-5856

\section{Editore}

Rosenberg \& Sellier

\section{Edizione cartacea}

Data di pubblicazione: 1 avril 2010

Paginazione: 200-201

ISSN: 0039-2944

\section{Notizia bibliografica digitale}

Luigia Pattano, «Édouard Glissant, Philosophie de la Relation», Studi Francesi [Online], 160 (LIV | I) | 2010, online dal 30 novembre 2015, consultato il 08 janvier 2021. URL: http://journals.openedition.org/ studifrancesi/7402 ; DOI: https://doi.org/ERREUR PDO dans /localdata/www-bin/Core/Core/Db/ Db.class.php L.34 : SQLSTATE[HY000] [2006] MySQL server has gone away

Questo documento è stato generato automaticamente il 8 janvier 2021.

\section{cc) (†) $९$}

Studi Francesi è distribuita con Licenza Creative Commons Attribuzione - Non commerciale - Non opere derivate 4.0 Internazionale. 


\title{
Édouard Glissant, Philosophie de la Relation
}

\author{
Luigia Pattano
}

\section{NOTIZIA}

ÉDOUARD GLISSANT, Philosophie de la Relation, Paris, Gallimard, 2009, pp. 161.

1 A quasi due decenni di distanza dal celeberrimo e fondamentale Poétique de la Relation (Paris, Gallimard, 1990), vero e proprio cuore del pensiero glissantiano, l'autore pubblica ora un testo dal titolo sorprendente, per non dire spiazzante, per chi conosce la sua ritrosia verso ogni tentativo di sistematizzazione. Philosophie de la Relation sembra difatti annunciare un cambiamento, o meglio un ampliamento e un rovesciamento di prospettiva: la scelta di sostituire il termine "poetica» con quello comprensivo di «filosofia» lascerebbe presagire il tentativo di far confluire la poetica della Relazione in un campo più ampio, più strutturato e più rigido dal punto di vista lessicale e formale. $\mathrm{E}$ tuttavia, se il titolo potrebbe trarre in inganno, il tono e lo stile del testo risolvono l'ambiguità: non ci troviamo di fronte a un ripensamento, ma all'ennesimo tentativo di fissare e precisare i concetti-chiave di un pensiero che si vuole palesemente sfuggente, «errante» come direbbe il suo autore, nel suo rifiuto della trasparenza.

Intitolata ermeticamente «Souques», la prima sezione del libro è dedicata alla poesia, la cui genesi risale, secondo l'autore, all'origine del mondo, all'epoca in cui tutto era ancora indistinto e tra gli uomini non esistevano differenze. Nata da una parola sacra che aveva desiderato in un primo tempo prevenire o combattere il processo di differenziazione, quindi far convergere le distinzioni in un tutto, la poesia è associata, nell'immaginario del poeta, alla vecchia casa natale, la «maison primordiale» ch'egli aveva cercato, dopo molti anni di assenza, di ritrovare assieme al figlio. Due oggetti, la casa e la poesia, appartenenti a mondi diversissimi e accomunati, tuttavia, da un medesimo destino: l'essere scomparsi, a un tratto, nelle profondità della terra. I dati autobiografici si (con)fondono qui come altrove, nelle pagine più riuscite, più ispirate $\mathrm{e}$ 
ammalianti, con le intuizioni dell'autore sul componimento poetico, «la seule dimension de vérité ou de permanence ou de déviance qui relie les précences du monde» (p. 19).

Che il testo si apra su queste considerazioni "poetiche" (nel duplice senso di intrise di poesia e aventi la poesia come oggetto) non è casuale né privo di interesse. Oltre a mostrarne la pervasività, Glissant elegge difatti la poesia a insostituibile forma di conoscenza e indica chiaramente una direzione, un modo di procedere che ha più $\mathrm{i}$ tratti dell'esplorazione poetica che del discorso filosofico comunemente inteso, più della «passe» che del «chemin» - potremmo dire usando alcuni vocaboli familiari ai suoi lettori. E allora perché parlare di filosofia proprio ora, ribattezzando così la propria teoria a questo punto di un percorso intellettuale caratterizzato da una notevole prudenza lessicale? O per dirla con le parole dell'autore stesso: «qu'est-ce que ainsi, une philosophie de la Relation?»(p. 82). La domanda giunge all'incirca a metà libro, all'interno della seconda sezione, elaborata a partire da alcune conferenze tenute da Glissant nel 2008. «Phases» (è così che si intitola questo capitolo corposo) si presenta come il sunto e la ripresa degli enunciati fondamentali contenuti in un altro suo testo, cui si richiama esplicitamente: Une nouvelle région du monde (2006). Dopo aver passato dunque in rassegna alcuni concetti della sua poetica (archipel, tremblement, frontière, errance, créolisation, imprévisible, opacité, Relation, trace...), l'autore avanza una risposta: «Un impossible, en tant qu'elle ne serait pas une poétique». E, a seguire, una definizione di filosofia: «un système non systémisé de tant de données aux conjonctions si dramatiques: la vitesse, les inextricables, cet inattendu, nous attrapent, nous transportent». Comprendiamo dunque che Glissant parla (a dire il vero non molto) in questo testo di «filosofia» pensando alla sua poetica: giacché solo la poesia è in grado di dire l'infinita diversità del Tout-Monde, «c'est-à-dire une totalité non totalitaire» (p. 112), le poetiche - afferma - rappresentano l'unica possibilità di accostarsi al tutto consentendo al tempo stesso di liberarsi dalle visioni globali, «ou des orbes de synthèse, qui nous engonceraient dans cette illusion que nous maîtrisons le chaos du monde» (p. 83). Ogni poetica è invece «un premier abord, une inscription rêche, dans l'inextricable» (idem). Provvisoria e incerta, errante.

4 Sulla nozione di «incerto», che non è «incertezza» bensì «frontière du vécu au pensé» (p. 97), si apre «Casses», terza parte del libro, dedicata a una peculiarità irrinunciabile della Relazione: la differenza. «Signe et relais des unités-diversités» (idem), la differenza concorre alla fusione e insieme alla distinzione, ed è «avant tout la conductrice de cette quête, par quoi l'écho égaré de la rivière ou la ruine enfouie de la case renaissent au chant: une composante, un relais, une liaison de ce qui persiste» (p. 102).

5 Nella quarta sezione («Erres»), Glissant ritorna con il pensiero alla vecchia casa del morne Bezaudin, viaggio che lo conduce dapprima a una riflessione cosmica, quindi alla rievocazione del percorso di Mathieu Béluse nella Lézarde (1958), infine al ricordo di quel momento epocale che fu il Congresso parigino di artisti e scrittori neri del 1956. Parole di riconoscenza sono riservate ad Alioune Diop, una delle anime, insieme ad Aimé Césaire, dello storico congresso. Del celebre poeta-sindaco l'autore ricostruisce invece il percorso intellettuale ed elogia l'opera letteraria. Quella di Césaire - afferma Glissant - è una poetica di vulcani ed eruzioni, «déchirée des emmêlements de la conscience, parcourue des flots déhalés de la souffrance nègre, avec parfois une surprenante tendresse d'eau de source, et partout de boucans de joie et de liesse» (p. 133). 
6 In «Vires», l'autore ritorna all'antico morne di Bezaudin dove la vista di un albero che non sa nominare lo spinge a interrogarsi sulla questione del nome. Le poetiche, ci dice, inducono da un lato a nominare con intelligenza, ma dall'altro suscitano anche l'oscuro desiderio del nome impossibile, «ou à vivre un désir impossible du nom» (p. 141). Il pensiero va quindi dapprima alla Pelée, alle sue tracce nell'immaginario martinicano, poi alla madre e al momento in cui Glissant ne aveva scoperto il nome per esteso: Adrienne Marie Euphémie. Scoperta che aveva forse sollecitato il giovane poeta a leggere nel nome materno - «Ce nom d'Euphémie, qui est si rare et si beau, et qui sans doute avait été dévolu au gré du calendrier ou de la fantaisie d'un parent ou du sarcasme d'un commis d'état civil» (p. 144)-le dinamiche della Relazione, una sua logica sotterranea. Il ricordo della madre lo porta al cimitero e ad alcuni luoghi della sua isola, di cui Glissant enfatizza ancora una volta l'importanza. Il luogo - ripete - è da intendersi non come entità isolata, particolare, bensì nella relazione con il mondo cui anela giacché esso è sempre condiviso ed è solo a partire da esso che è possibile intuire il Tout-Monde.

7 Prima di chiudere il testo con un omaggio in versi al celebre poeta palestinese recentemente scomparso Mahmoud Darwish e un breve passaggio dedicato alla «Végétation de l'acoma», Glissant ritorna all'espressione "philosophie de la Relation» offrendocene un'altra definizione. «La philosophie de la Relation»-ci dice-«est l'aspiration fragile, l'autre lieu où surprendre ces manières multipliées que nous avons de partager nos rapports rhizomiques, sans qu'elle confonde jamais ces manières les unes dans les autres, et sans que leurs différents, dont les opacités déjà correspondent, soient opposés en absolu, fut-ce en une fois» (p. 148). Un modo assai poetico ed elusivo che sembrerebbe di fatto volto a non definire, di certo a non circoscrivere, quest'uso personale, e del tutto inconsueto per chi come Glissant ha una formazione di filosofo, del termine filosofia. E rivelatore di un modo di procedere che finisce per mettere a disagio il lettore, facendolo sentire inadeguato di fronte a un pensiero che si arroga il diritto di essere opaco ed evasivo.

8 Se il pregio letterario di questo e degli altri testi teorici di Glissant è indiscutibile, una tale tecnica potrebbe far insorgere qualche sospetto circa la validità epistemologica di una teoria che pare non voglia mai essere del tutto compresa. Come si evince dallo strutturarsi dei capitoli, Philosophie de la Relation è un testo ibrido sia dal punto di vista della forma che del contenuto. Un testo che, malgrado il titolo e l'impianto teorico, non manca di guizzi poetici e di riflessioni intimistiche. Se questo non può che essere annoverato tra le sue qualità, la perplessità per la scelta del termine filosofia resta, avvalorata dall'incertezza con cui lo definisce il suo autore, insieme a un certo disagio nei confronti di un pensiero che sembra difficilmente criticabile. 